\title{
Identification of Radio Network Routing Method Based on Genetic Algorithm
}

\author{
Shuguang $\mathrm{Fu}$ \\ Qingdao Vocational and Technical College of Hotel Management \\ Zsbqchm@126.com
}

\begin{abstract}
As one of the most effective measures to solve the lack of spectrum, identification of radio technology is becoming more and more attention by the people, a large number of scholars and research institutions devoted to energy to study. For identification of multi-channel characteristics of radio network, extend multiple channel interface structure model, node model and using expanded NS2 network simulation tool for the proposed capacity satisfaction and link stability for routing metrics routing protocol based on genetic algorithm and simulation results proved that the proposed algorithm has good performance and usage of the group can pass spontaneously, but slightly on the routing cost.
\end{abstract}

Keywords: identify radio network, genetic algorithm, the routing protocol, NS2

\section{Introduction}

With the rapid development of wireless communication technology, the shortage of radio spectrum resources is to limit the bottleneck of sustainable development and application of wireless mobile communication, resource scarcity has become increasingly serious, to the new system, the frequency spectrum of the business and technology are very low. Spectrum is a limited, precious and nonrenewable resources, in the spectrum resources under the background of the increasingly aggravation of the contradiction between supply and demand, identify radio technology arises at the historic moment. [1] With the existing spectrum management and distribution strategy is a kind of static, identify radio technology in theory allow multidimensional in time, space and frequency spectrum of reuse, can realize the reliable communication, is regarded as one of the top technology in the next generation of wireless communication.

Identify radios are effective measures to solve the frequency spectrum is scarce, which identify users by using spectrum is obtained by means of perception, the authorized users, secondary utilization of unused spectrum to identify radio network routing presents the new characteristics of contrary to the wireless network, such as diversity and time-varying spectrum set with nodes are available, and more dynamic network topology, only consider the hop routing possible because the link capacity and stability of the routing delay in consumption big problem. In this case, the identification of radio network routing selected faces new problems and challenges, to have new requirements.

Service level has been a hotspot of research on network, in the network layer is the transmission must be satisfied by the quality, meet two or more independent of each other routing problem is a selected parameter constraint network processing problem completely. [2-3] In the identification of radio network, the node of the link between dynamic big lead to alternative routing performance gap between larger, for a high performance routing selected "best" will the traditional network routing optimization strategy of selected into "best". Genetic algorithm is a global random search algorithm, the genetic algorithm is used to improve routing selected routing protocol will effectively solve the above two questions. 
Based on the above discussion, to identify radio routing algorithm and protocol of the study is very necessary, the routing problem in this paper is aimed at identifying the user, due to its spectrum is discrete, identify the network routing design in addition to the problems, such as to consider the hop to consider spectrum characteristics and stability of the link. This paper applies genetic algorithm into the routing selection, then to improve the on-demand routing protocols make it suitable for identification of radio network and routing strategy of this paper, based on more network topology information, selected the existence of high performance routing.

Routing technology is the key technology of the network layer, as well as the whole service level of network security. Recognition in the radio system users and the authorized users. Users will be able to use the authorization of free spectrum recognition, but after the rise of authorized user must exit this spectrum. Based on this mechanism, compared with the traditional network of fixed frequency, identification in wireless Ad Hoc network frequency change with time and space. [4-6] The radio environment identification of routing algorithm and protocol faces new problems. Is due to the dynamic spectrum, the connection between the nodes situation changes, compared with traditional network identification of radio network has the characteristics of high dynamic routing, if the routing for a link with the appearance of authorized users, breaking probability is too large, will greatly reduce the performance of routing, this is cannot be ignored in the routing protocol, this is not involved in the traditional routing protocols. As far as possible in order to conform to the requirements of the business and even meet the demand of business service level, we must first consider the spectrum in the routing technology performance and the influence of the use right by, in addition to the traditional based on hop count, need to introduce a new measure to improve the recognition performance of network routing.

Routing algorithm is the soul of the routing protocol, the purpose is to find a best routing performance. In identifying the link quality change is big, between the nodes in the source node to the destination node of the performance gap between alternative paths. In traditional routing protocol routing discovery mechanisms, ignores many potential routing, just do the routing performance of "the best", it is concluded that the routing is not necessarily the best. Routing request in order to meet the demand of the service level of the business, as far as possible need right by the information processing and find out that exist in the network performance better path, therefore routing algorithms and protocols have larger research space.

In this paper, based on Ad Hoc network, to identify the characteristics of the radio spectrum dynamic change is big comprehensive consider whether the bandwidth of the available spectrum and the available spectrum stability by the influence of selected, put forward the routing metric: channel capacity satisfaction and link stability. In routing selected using the routing selection algorithm based on adaptive genetic algorithm to solve the routing of demand service level performance, and make corresponding improvement in the traditional DSR protocol, the algorithm can find out the high performance of the network routing. According to the different recognition radio network environment to adapt to the selection of adaptive routing strategy. Strategy is largely based on service level Ensure and load balancing. When the network overall load lighter, routing mainly to meet the business needs of parameters such as bandwidth and delay; When the network load is heavier, the routing is focused on load balancing. The strategy adjustment way is mainly on the weight of the routing metric, the method of the whole network information demand is bigger.

\section{Related Works}

\subsection{Identify Radio Network}

If you identify radio technology is applied to actual wireless communication, the first 
consideration is the network mode. Networking refers to the interaction between multiple nodes, as a whole has the ability of communication. Used to identify radio technology node can independently or jointly with the existing network of radio network [7-8]. Different network structure has different function, also have different requirements on the layers of communication technology. For the network layer routing technology is the core technology, the design direction and to achieve the goal of often were different according to different network architecture. Identify radio network architecture mainly has three kinds: intensive network architecture, a distributed network architecture and mixed type network architecture.

In the intensive network, all users need to listen to scheduling and distribution center controller, the controller in the center of the communication needs to apply for the channel, IEEE. 802.22 a typical representative of the intensive type is to identify radio network, because this kind of network topology's center controller, so easy to manage, but the center controller to identify the stand or fall of performance and load ability of high and low also determines the intensive network architecture to identify the stand or fall of network performance.

Another network mode distributed network architecture, the user randomly dispersed in the network, with the method of Ad Hoc, composed of a decentralized identification of wireless network, when a user to communicate, because there is no central controller to manage network resources, each user according to their own spectrum perception and the study of environmental parameters, the selected channel and transmission parameters signal in the form of single hop or multiple hops. This way, the network is convenient, but also because of big topology dynamic security network performance has the certain difficulty. If a local emergency within the network, will not affect the global. Under the distributed network architecture, the user need to competition of network spectrum, the routing selection in terms of suppression interference and maintain stability of the link has the certain difficulty, packet switching process is relatively complex, makes the transmission performance guarantee is difficult.

The current wireless network, the frequency spectrum resource management using the authorization and unlicensed spectrum distribution system, this system is static, managed by government agencies and division, such as television and radio frequency can only be used by its authorized users. Emerging with the rapid development of wireless communication, network business types of proliferation makes the industrial, scientific and medical band have crowded, the unauthorized frequency band is very rare. From the point of view of time and space, the use of spectrum is very unbalanced, low-frequency especially transmission performance better global authorized spectrum utilization rate at low levels, rather than the authorized spectrum is becoming saturated.

Due to identify radio network has the characteristics of high dynamic, so network architecture can't effectively adapt to the characteristics of the intensive type, only under the condition of the spectrum change relatively small intensive network to embody the advantage. Distributed network flexibility and self organization, so the researchers than is generally believed that this kind of network architecture intensive network is more suitable for the identification of radio systems. After the combination of the intensive type and decentralized control manner have mixed type network architecture, such as identification of Mesh network is relative to the intensive type network architecture, network architecture control more complex mixed type, but the reliability has been improved greatly.

In this article to study the identification of radio network is based on Ad hoc network of networks in more jump way. This is a kind of identification of a decentralized network, does not require network infrastructure support, each node in the network can be awareness of idle spectrum, apply to temporary communications, emergency communication, etc. Currently, identify radio routing at the exploration stage, various research results has its limitations, identify the network routing performance improvement space, has a great deal of research study is suitable for the high security routing identify radio is also very 
necessary. In recent years in terms of Ad Hoc network routing selection algorithm, based on the intelligent optimization algorithms of Ad Hoc protocol got the favour of many scholars, the genetic algorithm, ant colony algorithm and particle swarm optimization (pso) algorithm and other intelligent optimization algorithms.

\subsection{Genetic Algorithm}

Genetic algorithm is based on natural selection and genetic mechanism of the theory of optimization method, which simulates the biological evolution in nature, is a kind of efficient parallel global search method. It to the idea of "survival of the fittest" as the center, USES the evolution laws of nature, the out the individual difference, keep good individual genetic, it adaptively control the searching process to get the optimal solution, the algorithm of the whole operation process according to the evolutionary mechanism of the organism, starting from a random group, according to the situation of the environmental fitness "natural selection" excellent individuals to retain, and carries on the breeding, derived the next generation, a generation of inherited the good genes of the parent group, has a better performance, also may produce gene mutation in the breeding, genetic down from one generation to another. Genetic algorithm in computing the abstract process of organism evolution process, the problem of parameter space, replace with coding space, according to select individual fitness function, crossover and mutation operation for individuals in the group, resulting in a new species, forming an iterative process. Overall, the new generation of group received the evolution, constantly updated, continuous development, improve the ability to adapt to nature, to promote population evolution in the direction of optimization, and finally find out the optimal solution.

Genetic algorithm can effectively solve the problem of belongs to network processing completely, some scholars also apply it to the routing problem, selected routing is the network service level to tackle the problem of completely in some complicated cases it is difficult to find the optimal solution, seek out the satisfied solution in the routing is in conformity with the actual, and genetic algorithm is one of the best tools. Introduces genetic algorithm and on-demand routing, routing selection when the genetic operation is actually a mining of network information processing, get better performance of the available potential routing [9-10].

According to the working process of genetic algorithm, the basic genetic operation steps for the following steps:

a)Genetic encoding in solving specific problems, it must be the target problem into the chromosome bit string of genetic algorithm, at the same time be able to chromosomes represented as the actual problem, namely to determine the encoding and decoding. To determine the optimization problem, a set of individual genetic phenotypes of space known as the problem space. Genetic algorithm has strong robustness, general issues gene can be used in one dimension of fixed-length chromosome representation. Genetic encoding binary encoding, sequence code, tree coding, etc., is one of the most basic binary code.

b) Calculate the fitness function. In order to implement the principle of survival of the fittest, must the adaptability evaluation of individual bit string. The fitness of an individual for the environment, is a kind of be used to measure the degree of individual fitness in a population parameter values, it is usually in the form of a fitness function value. So the individual fitness value of high and low, directly reflects the individual's survival ability strong and the weak in this environment. In general, if the chromosome bit string of genetic structure is good, then the individual will have higher adaptive function value, indicates that the individual can obtain higher value, have stronger ability to survive.

c) Genetic factorsIn the genetic operations, the whole process is simulated in the process of natural selection and genetic mutation breeding, hybridization and wait for a phenomenon, so as to realize the evolutionary process of evolution. In general, genetic 
factors are selected, three kinds of crossover and mutation.

1) The Selected Factors

Selected, that is, from the current group of selected individuals to generate high fitness value, the mating pool. It is "the fittest, survival of the fittest" simulation of natural evolution principle. Low fitness of individuals in the smaller probability genetic into the next generation of group, the high fitness individuals with larger probability genetic to the next generation. In fact is to determine in what way from the parent population from which the individual genetic group, a genetic algorithm to the next generation. At present there are mainly selected by adaptive value proportion, sorting, selection, league and selected elite selection etc.

2) Interleaving

In biological genetic and evolutionary process, mating restructuring is an important link in genes, determine the direction of evolution and features. Interleaving is imitating nature of genetic algorithm genetic recombination process of sexual reproduction, according to a certain way for part two pairs of chromosomes swap genes, and thus form a new individual. Its role is to pass the original good genes to the next generation of individuals, and generate contains more complex genetic structure of the new individual. Groups within each individual random collocation in pairs, a pair of chromosomes in one or a few genes with a certain probability exchanging position, generating new individuals.

\section{3) Mutation factor}

Mutation factor is used to simulate the nature evolution genetic algorithm one gene locus on a chromosome mutations occur. In genetic algorithm, the mutation operation is based on a certain probability randomly take out from the group of a number of individuals, and then randomly selected individuals of a particular for mutation operation. In order to guarantee the stability of population development, general mutation probability values is smaller. Introducing mutation factor in genetic algorithm, on the one hand, can make to maintain the diversity of population in the process of evolution, to prevent "premature" phenomenon; On the other hand can improve the local search ability of genetic algorithm.

d) Group setting and initialized. Genetic algorithm was conducted on the solution group, and group set is fundamental for the performance of genetic algorithm. The diversity of the individuals in the population size, the larger the group, the more rich, the algorithm into the smaller the risk of local solution, but with the increase of population size, amount of calculation is also will increase significantly. If the population size is too small, can make the searching space of genetic algorithm is restricted, may produce "premature" phenomenon.

e) Control parameters. Genetic algorithms in the process of operation, has a set of parameters have significant effects on its operation performance, need to be selected properly and control, which makes the search of genetic algorithm to achieve the best performance. To the selected operation parameters in genetic algorithm are mainly population size, crossover probability and mutation probability.

The service level of the cognitive radio network routing algorithm is described in the process of involves many variables,

For the convenience of narrative, of these variables are defined as follows:

(1) Cognitive Ad Hoc networks can be expressed as directed graph $X=(A, F)$, among them $\mathrm{A}$ for cognitive node set, $\mathrm{F}$ is A collection of links between nodes.

(2) Because there are a of each cognitive nodes in the network of maximum transmitted power is limited, so remember to $\mathrm{P}$, its biggest transmission range for $\mathrm{T}$. 
(3) The distance between nodes $v_{i} 1$ and $v_{j}$, if $l_{i k}<R$ the node has one path may be between 1 and 2 .

For a routing request, one of the most ideal state is one who can find a from the source node and destination node $\mathrm{d} s$ path between $\mathrm{p}$ said path in the $\mathrm{p}$ ( $\mathrm{r}$ as a link), not only has the minimum routing cost, but also can meet the requirements of the service level at the same time (three routing restrictions: here we assume that the time delay, delay jitter, packet loss rate), satisfies the requirement of the biggest packet loss rate $Y_{\max }$, meet the requirements of the end-to-end delay $U_{\max }$, satisfies the requirement of end-to-end delay jitter $\varepsilon_{\max }$. The most ideal state can be shown by the following optimization problem to describe.

The listed optimization problem as follows:

$$
\min f
$$

The corresponding constraint conditions for:

$$
\begin{gathered}
Y_{v}=1-\prod_{e-p}(1-Y(r)) \leq Y_{\max } \\
U(p)=\sum_{e-p} U(r) \leq U_{\max } \\
\varepsilon(p)=\sum_{e-p} \varepsilon(r) \leq \varepsilon_{\max }
\end{gathered}
$$

It is obvious that service level routing problem can be described as a constrained minimum optimization problem.

\section{Improved Recognition Radio Routing Protocol Based on Genetic Algorithm}

Routing is usually contains two parts: routing and forwarding. Choose the way determine the best path to reach the destination node; Forward the best path along the selected transmit information packet. Accordingly, routing protocols can be divided into the routing protocol and routing forwarding selected. Routing protocol and routing forwarding selected is the concept of both cooperate with each other and are independent of each other, the latter maintain routing tables, using the former the former to take advantage of the latter at the same time provide function to distribute routing protocol data packet. Mentioned in this article the routing protocol that is the way by the selected protocol [12].

Traditional wireless network has a lot of mature routing protocol, the front have been mentioned. But the traditional Ad Hoc network routing protocol is not suitable for identification of radio network, but can be used to draw lessons from. Identify radio routing protocol can continue to use the basic process of traditional Ad Hoc network routing protocols, the change can be in a different part of the algorithm to adapt to the requirements of the network, can also add various nodes in the original message format spectrum information or other related information. RREQ and RREP messages carry the source node and destination node and intermediate node spectrum information, together they can provide a full spectrum using the information on the path. Combined with a spectrum allocation in the routing selection.

Traditional DSR routing protocol, when selecting the optimal routing path, only to the hop count as the basis of the selected route, selected by a minimum of at least, that is, the hop routing nodes. This is based on the minimum hop routing algorithm has great limitations in the identification of radio. And in the general Ad Hoc network routing 
protocol by the request packet forwarding only once, the destination node routing selected limited, waste a lot of good potential routing performance. In recognition, under the new characteristics of network path is a big gap between the performance, in order to meet the general business route requests even have business service level requirement, this paper puts forward to link capacity and link stability measure routing selection strategy based on genetic algorithm.

Protocol used on demand type as the DSR routing protocol process framework, divided into the routing request, routing selection, routing and routing maintenance sections response and satisfaction to capacity and the path stability as the optimization goal, at the destination node execution algorithm. On the basis of the DSR protocol to make the corresponding improvement. Make it suitable for recognition of radio model, also can find the path of the high performance through genetic algorithm.

In this paper, we study the recognition network is distributed Ad Hoc network, in which all nodes communicate through multiple hops. The following is a network of assumptions, routing protocol based on the following conditions:

(1) All tell the link between nodes are bidirectional.

(2) Identify radio in the network nodes are stationary or slow moving quasi static.

(3) Each node has a spectrum detection ability.

(4) Between each node in the network are synchronous, there is a public control channel between nodes.

(5)Each node has a transceiver and a receiver.

Deal with on-demand routing protocols DSR, on the basis of the main process of the protocol message information and route discovery mechanism to make the corresponding improvement to adapt to identify without wires. The main routing discovery protocol, selected, response and repair mechanisms are described in detail, has laid a good groundwork for the subsequent algorithm simulation.

\section{The Simulation Results and Its Analysis}

Used for NS2 simulation tool, the English always referred to as a Network Simulator Version 2, NS2 is a free software, at the same time can run on Windows and UNIX systems, its all source code is open, study and extension of Network is very convenient, it is also a more academic problems by using NS2 to simulate Network. Based on the NS2 as a simulation tool is the main reason of NS2 scalability and flexibility [13-15].

Using NS2 environment on the routing protocol performance simulation in this paper, in a $1000 \mathrm{~m}$ by $1000 \mathrm{~m}$ area placed several identify nodes, motionless as already assume that all nodes. Transmission range of each node is $250 \mathrm{~m}$, in $40 \mathrm{MHZ}$ to $2.0 \mathrm{GHz}$ spectrum range set 5 channel, each node randomly selected from 3 to 4 spectrum form SOP set to simulate the dispersion spectrum of scenarios, channel bandwidth is $3 \mathrm{MHZ}$, according to channel ON - OFF model, each channeloccupancy time parameter ${ }_{X_{m}}$ random value between $0.5 \sim 0.7$, free time parameter $Y_{m}$ randomvalue between $0.2 \sim 0.6$, the reference time T take 12 seconds. The MAC layer using standard IEEE802.11 DCF model. Routing metric weights $a=b=0.6$. Selected randomly in the process of the whole simulation source node and destination node injection several routing connection request, the averaged time interval is set to $15 \mathrm{~ms}$, business types for CBR data flow, data flow rate of $2.5 \mathrm{Mbps}$. The simulation time of $150 \mathrm{~s}$.

The population size selected 25 of genetic algorithm, the initial mutation probability $Z_{m i}=0.6$, the minimum mutation probability network processing $Z_{m \min }=0.3$, the initial crossoverprobability $Z_{c i}=0.5$, the smallest crossover probability network processing $Z_{c i \min }=0.6$, termination of algebra selected 60 generations. The actual usage, the packet 
transmission rate and routing cost three performance indicators to the simulation in this paper, the performance of the algorithm agreement.

The actual usage: unit time through the network system in the actual amount of useful data. It can reflect spectrum bandwidth utilization ability, is an important indicator of overall performance reflects the network, in does not affect the other performance under the premise of the network protocol design is often to maximize the use of as the goal.

Packet delivery ratio: destination node group received total number and the ratio of the total number of source node sending packet, also reflects the packet loss rate, to a certain extent, depicting the validity of the agreement and transmission quality.

Routing consumption: refers to the network transmission of control packets of all communication and transport package of the ratio of the number. The indicators reflect the effectiveness routing protocol.

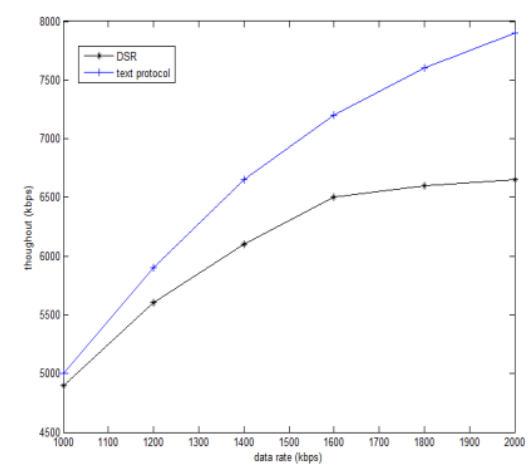

Figure 1. The Throughput of Different Flow Rate

Figure 1 shows the different data flow rate, the actual usage of performance of the network, the node number of 50, and the whole simulation of five data flow for communication, data flow rate from MKBPS change 1 to 2 MBPS. You can see that the growth of actual usage of the two kinds of agreement does not with the improvement of data flow rate and linear growth, because the network channel resource is certain, the resource utilization in reaches a certain degree will slow growth trends, which tends to be stable. Can also see from the diagram, when the data flow rate is small, two kinds of protocol can be normal work, the use of difference is not obvious. When data flow rate increases, the business requirement for channel capacity is bigger also, in this agreement fully consider when routing selected channel capacity satisfaction rate and the link stability, so the selected path has a better average capacity, makes the usage very obvious advantage than the DSR protocol. Simulation experiments show that the protocol has better performance of actual usage.

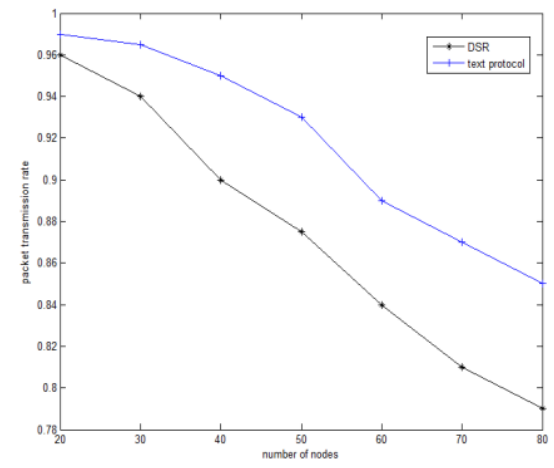




\section{Figure 2. The Packet Delivery Ratio of Different Number of Nodes}

Figure 2 shows that with the increase of number of nodes, two kinds of protocol packet transmission rate changes. You can see two Figure 6.5 shows that with the increase of number of nodes, two kinds of protocol packet transmission rate changes. It can be seen that both agreement transfer rate show a downward trend. This is because with the increase of the number of nodes, routing requests jumping increase in the number of the selected path, makes the routing increases the probability of disruption by spectrum, link disconnect lead to packet loss and packet loss. But as the number of node size bigger, in this paper, protocol packet transfer rate is significantly better than the DSR protocol, because in this paper, the routing of the agreement the selected considering the link stability, more number of nodes in the network based on the genetic algorithm can better dig out the network stability in a better path, give priority to the selected relatively stable channel grouping, relatively reduced the interrupt probability routing, makes the routing is stable. The DSR protocol in the link between randomly selected channel communication, on the routing outage probability is bigger, so the packet loss. Can be concluded in this paper, from the analysis of simulation results and the performance of the agreement on the packet transmission rate are better than the DSR protocol.

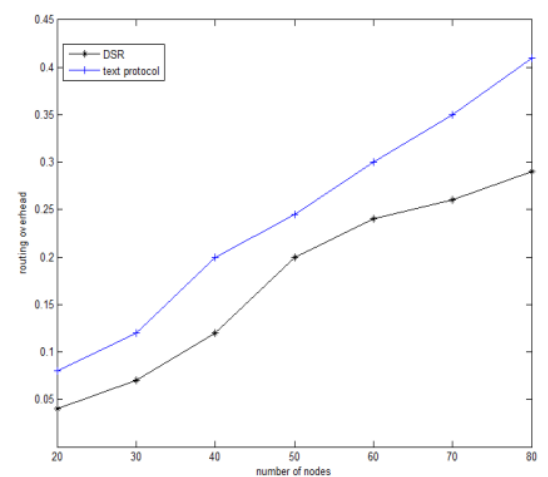

Figure 3. The Routing Overhead of Different Number of Nodes

Figure 3 shows the routing cost along with the network node number change. Routing protocols use is also a measure of a routing protocol is practical as can be seen from the diagram in this paper, the agreement on the node consumption was a little higher than the DSR is relatively small, as the number of nodes increases, the scale of network is more and more big consumption of this agreement will be significantly higher than that of the DSR protocol. Because this agreement in order to find more potential routing to start the genetic algorithm, the same node of RREQ can handle three times, originally the DSR protocol of RREQ processing only once, so cause the increase of consumption of the routing. But in the network scale is small because the routing protocol in this paper the interrupt rate lower than the DSR protocol, consuming less than the DSR routing reconstruction, so with the consumption of the DSR protocol. But in a larger network routing protocol in this paper the control packet increased consumption led to a rapid growth. Although protocol in this paper, the improved algorithm on the usage and the packet transmission rate has a better performance, but because genetic algorithm need to accumulate certain RREQ, collect more network topology information to effectively implement, so that the routing cost is greater than the DSR protocol. So according to the specific requirements of the size of the network and routing business, to the limitation on the number of control packets for specific is the place that can improve in this agreement, to consider a single path of folding performance and the network overall performance. 


\section{Conclusion}

This paper describes in detail the NS2 nodes in the structure of the model. Then according to the characteristics of radio network to identify multi-channel extend NS2 nodes in the single channel of the channel structure model, at last, through the expanded NS2 network simulation tools for the proposed capacity satisfaction and link stability for routing metrics routing protocol based on genetic algorithm simulation, the results proved that the presented algorithm has very good usage and grouping can pass spontaneously, but on the routing cost is greater than the DSR protocol.

\section{References}

[1] N. Devroye, M. Vu and V. Tarokh, "Cognitive radio networks", Signal Processing Magazine, IEEE, vol. 25, no. 6, (2008), pp. 12-23.

[2] Y. C. Liang and Y. Zeng, PehECY, "Sensing-throughput tradeoff for cognitive radio networks", Wireless Communications, IEEE Transactions on, vol. 7, no. 4, (2008), pp. 1326-1337.

[3] I. F. Akyildiz, W. Y. Lee and M. C. Vuran, "A survey on spectrum management in cognitive radio networks", Communications Magazine, IEEE, vol. 46, no. 4, (2008), pp. 40-48.

[4] N. Nie, "Comaniciu C. Adaptive channel allocation spectrum etiquette for cognitive radio networks" Mobile networks and applications, vol. 11, no. 6, (2006), pp. 779-797.

[5] G. Ganesan and Y. Li, "Cooperative spectrum sensing in cognitive radio networks" New Frontiers in Dynamic Spectrum Access Networks, 2005. DySPAN 2005. 2005 First IEEE International Symposium on. IEEE, (2005), pp. 137-143.

[6] F. Saito, S.A. Moore and R. Barresi, "Unique role of dystroglycan in peripheral nerve myelination, nodal structure, and sodium channel stabilization" Neuron, vol. 38, no. 5, (2003), pp. 747-758.

[7] G. Molle, J. Y. Dugast and G. Spach, "Ion channel stabilization of synthetic alamethicin analogs by rings of inter-helix H-bonds", Biophysical journal, vol. 70, no. 4, (1996), pp. 1669-1675.

[8] S. A. Seoh and B. D. Gramicidin, "Tryptophans mediate formamidinium-induced channel stabilization", Biophysical journal, vol. 68, no. 6, (1995), pp. 2271-2279.

[9] E. Partheniades and R. E. Paaswell, "Erosion of cohesive soil and channel stabilization", Dept. of Civil Engineeering, State University of New York at Buffalo, (1968).

[10] M. Bansal, R. Rajput and G. Gupta, "Mobile ad hoc networking (MANET): Routing protocol performance issues and evaluation considerations", The Internet Society, (1999).

[11] T. Clausen, P. Jacquet and C. Adjih, "Optimized link state routing protocol (OLSR)", (2003).

[12] K. Sanzgiri, B. Dahill and B.N. Levine, "A secure routing protocol for ad hoc networks[C]", Network Protocols, 2002. Proceedings.10th IEEE International Conference on. IEEE, (2002), pp. 78-87.

[13] K. Sanzgiri, B. Dahill and B. N. Levine, "A secure routing protocol for ad hoc networks", Network Protocols, 2002. Proceedings.10th IEEE International Conference on. IEEE, (2002), pp. 78-87.

[14] S. J. Lee, W. Su and M. Gerla, "On-demand multicast routing protocol in multihop wireless mobile networks", Mobile Networks and Applications, vol. 7, no. 6, (2002), pp. 441-453.

[15] S. Murthy and J. J. Garcia-Luna-Aceves, "An efficient routing protocol for wireless networks", Mobile Networks and Applications, vol. 1, no. 2, (1996), pp. 183-197.

\section{Author}

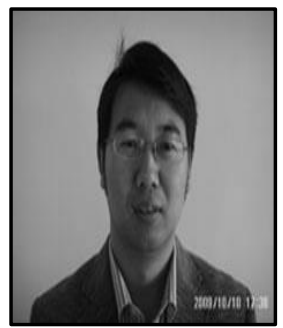

Shuguang Fu, received the Bachelor degree in Computer Engineering from Qingdao Technological University and Master's degree in Computer Engineering from China Ocean University, CHINA in 2004 and 2011 respectively. He is teaching and researching Routing Protocols at Qingdao Vocational and Technical College of Hotel Management. 